Schweiz. Z. Tuberk. 1961;18:I-IV

\title{
Contents, Vol. 18, No. 3, 1961
}

Klinisch brauchbare Einteilung

des Lungenemphysems

auf Grund der Resultate

von Lungenfunktionsprüfungen

Kolloquium, gehalten am VI. Internationalen Kongreß

für Erkrankungen der Thoraxorgane

des American College of Chest Physicians

in Wien am 29. August 1960

Herausgegeben von M. Scherrer (Bern)

\section{BASEL (Schweiz) S.KARGER NEWYORK}

Leitung Dr. M. Scherrer, Med. Univ. Klinik, Bern (Prof. W. Hadorn).

Teilnehmer

Dr. U. Bucher, Med. Univ. Klinik, Bern (Prof. W. Hadorn).

Dr. W. Hartung, Path. Institut der Univ. Münster (Prof. W. Giese

Dr. C.W. Hertz, Krankenhaus Tönsheide (Prof. J. Hein).

Prof. H. Julich, Med. Univ. Klinik, Halle (Prof. R.E. Mark).

Prof. N. G. M. Orie, Lungenabteilung Med. Univ. Klinik, Groningei

Prof. R. Rimini, Med. Univ. Klinik, Montevideo.

Dr. F. Schmidt, Krankenhaus Lainz, Wien (Prof. A. Sattler).

Prof. M.S. Segal, Tufts University, Boston City Hospital, Boston.

Dr. G.P. Sheldon, Presbyterian Medical Center, San Francisco.

INHALT

Vol. 18, No. 3(1961)

Einleitung. Von M. Scherrer, Bern 129

Das nicht obstruktive Lungenemphyßem 131

Von F. Schmidt, Wien; W. Hartung, Münster und M. Scherrer, Bern

Das obstraktive Lungenemphysem 140

Von G.P. Sheldon, San Francisco; W. Hartung, Münster; R. Rimini, Montevideo; M. Scherrer, Bern und M. S. Segal, Boston

Reversibilität und Irreversibilität des obstruktiven Lungenemphysems ... 159 Von N. G. M. Orie,

Groningen; M. Scherrer, Bern und U. Bucher, Bern

Unterteilungsmöglichkeiten des obstruktiven Lungenemphysems auf Grund

von gasanalytischen Untersuchungen 163

Von H. Julich, Halle; C.W. Hertz, Tönsheide; U. Bucher, Bern;

M. Scherrer, Bern; G. P. Sheldon, San Francisco und W. Hartung,

Münster

Schlußwort und Zusammenfasßung 184

Von M. Scherrer, Bern 